\title{
Disk mass and disk heating in the spiral galaxy NGC 3223
}

\author{
G. Gentile ${ }^{1,2}$, C. Tydtgat ${ }^{1,3}$, M. Baes ${ }^{1}$, G. De Geyter ${ }^{1}$, M. Koleva ${ }^{1}$, G. W. Angus ${ }^{2}$, W. J. G. de Blok ${ }^{4,5,6}$, \\ W. Saftly ${ }^{1}$, and S. Viaene ${ }^{1}$
}

\author{
${ }^{1}$ Sterrenkundig Observatorium, Universiteit Gent, Krijgslaan 281, 9000 Gent, Belgium \\ e-mail: gianfranco.gentile@ugent.be \\ 2 Department of Physics and Astrophysics, Vrije Universiteit Brussel, Pleinlaan 2, 1050 Brussels, Belgium \\ 3 Department of Solid State Sciences, Krijgslaan 281, 9000 Gent, Belgium \\ 4 Netherlands Institute for Radio Astronomy (ASTRON), Postbus 2, 7990 AA Dwingeloo, The Netherlands \\ 5 Astrophysics, Cosmology and Gravity Centre, Department of Astronomy, University of Cape Town, Private Bag X3, \\ 7701 Rondebosch, South Africa \\ ${ }^{6}$ Kapteyn Astronomical Institute, University of Groningen, PO Box 800, 9700 AV Groningen, The Netherlands
}

Received 5 November 2014 / Accepted 11 February 2015

\begin{abstract}
We present the stellar and gaseous kinematics of an Sb galaxy, NGC 3223, with the aim of determining the vertical and radial stellar velocity dispersion as a function of radius, which can help to constrain disk heating theories. Together with the observed NIR photometry, the vertical velocity dispersion is also used to determine the stellar mass-to-light $(M / L)$ ratio, typically one of the largest uncertainties when deriving the dark matter distribution from the observed rotation curve. We find a vertical-to-radial velocity dispersion ratio of $\sigma_{z} / \sigma_{R}=1.21 \pm 0.14$, significantly higher than expectations from known correlations, and a weakly-constrained $K$ s-band stellar $M / L$ ratio in the range $0.5-1.7$, which is at the high end of (but consistent with) the predictions of stellar population synthesis models. Such a weak constraint on the stellar $M / L$ ratio, however, does not allow us to securely determine the dark matter density distribution. To achieve this, either a statistical approach or additional data (e.g. integral-field unit) are needed.
\end{abstract}

Key words. galaxies: kinematics and dynamics - galaxies: individual: NGC 3223 - galaxies: structure

\section{Introduction}

The structure of galaxies and, in particular, their mass content and distribution have been extensively investigated in the past decades. Studying the mass distribution of galaxies is closely related to the disk mass problem: how does one associate the observed stellar light distribution to a certain stellar mass distribution? Typically this is achieved by scaling the stellar light distribution by a factor, the mass-to-light $(M / L)$ ratio, to derive the stellar mass distribution. However, the stellar $M / L$ ratio is not known a priori, and a number of routes exist for estimating it (De Jong \& Bell 2007; Courteau et al. 2014): for instance, via stellar population synthesis models (Bell et al. 2001; McGaugh \& Schombert 2014), spiral arms (Fuchs 2003), bar dynamics (Weiner et al. 2001; Pérez et al. 2004), and, most importantly for the present paper, using vertical velocity dispersions (Bottema 1997; Kregel et al. 2005; Bershady et al. 2010a; Martinsson et al. 2013).

These methods give only very roughly consistent results, and discrepancies can run up to factors of a few. A closely related issue is the maximum disk hypothesis: can the stellar disk contribution to the rotation curve be scaled up to its maximum possible value (van Albada et al. 1985)? Using the various methods described above to derive the stellar mass, authors have reached contrasting conclusions, ranging from close-to-maximum disk to submaximum disks (e.g. Weiner et al. 2001; Martinsson et al. 2013).

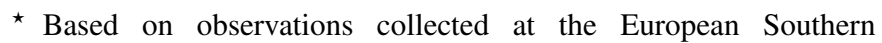
Observatory, Chile, under proposal 68.B-0588.
Determining the stellar mass is important for the derivation of the dark matter distribution in galaxies, notably because the shape of the dark matter density distribution is related to that of the stellar mass (de Blok et al. 2008). The dark matter distribution has been the topic of a debate in recent years, where it has become apparent that there is a discrepancy between the dark matter density profiles found in dark matter-only $\Lambda$ cold dark matter $(\Lambda C D M)$ simulations of galaxy formation and the observed dark matter density profiles (e.g. de Blok et al. 2001; Gentile et al. 2004; van Eymeren et al. 2009; Kuzio de Naray \& Kaufmann 2011). Recently, various models involving the (unknown) effect of baryons on the dark matter distribution have been proposed that try to reduce or remove the discrepancy between simulations and observations (e.g. Oh et al. 2011a; Governato et al. 2012; Macciò et al. 2012; Cloet-Osselaer et al. 2012; Di Cintio et al. 2014).

The internal kinematics of galaxies have been used to investigate a wide variety of topics, including the mass distribution of galaxies (e.g. Bosma 1981; Persic et al. 1996; Oh et al. 2011b; Gentile et al. 2013) and galaxy evolution, in particular disk heating (e.g. Minchev \& Quillen 2006; Gerssen \& Shapiro Griffin 2012; Sellwood 2014). A possible route for estimating the stellar mass is to use the relation between vertical stellar velocity dispersion and surface density, as done by the DiskMass survey team (Bershady et al. 2010a). Stellar kinematics can also be used to investigate the process called "disk heating", which is the increase in the random velocity of stars in the galaxy disk with time. By measuring the vertical and radial velocity dispersions, $\sigma_{z}$ and $\sigma_{R}$, one can test the validity of theories put 
forward to explain the disk heating phenomenon (Jenkins \& Binney 1990; Shapiro et al. 2003), e.g. spiral structure (Sellwood \& Binney 2002), scattering off giant molecular clouds (Hänninen $\&$ Flynn 2002), the effect of gas expulsion on compact embedded star clusters (Kroupa 2002; Candlish et al. 2014), or the infall of substructure onto the galaxy (e.g. Kazantzidis et al. 2009). There is still no consensus concerning the dominant mechanism(s) at play. Gerssen \& Shapiro Griffin (2012) find that the variation in $\sigma_{z}$ and $\sigma_{R}$ with Hubble type suggests that spiral structure might be the primary radial heating agent, while giant molecular clouds are consistent with being the 3D heating agent. These results are based on a handful of galaxies, and the values of of $\sigma_{z}$ and $\sigma_{R}$ are derived through a multi-parameter fit of the observed velocity dispersion and rotation velocity profiles. We use a similar approach here.

To simultaneously investigate disk mass and disk heating, as a pilot study we observed the Sb spiral galaxy NGC 3223, whose Hubble distance (corrected for Virgocentric flow) is about 38 Mpc. NGC 3223 was selected because of its southern declination (which made it accessible to observations with the VLT), its moderate inclination, and its relatively smooth optical appearance. It was part of the sample of 74 galaxies for which Palunas \& Williams (2000) made 2D H $\alpha$ velocity fields, and then derived a rotation curve. We obtained both HI data, to extend the rotation curve to larger radii, and stellar kinematics along the minor and major axes. NGC 3223 was also part of the sample of Kassin et al. (2006a,b), who used the rotation curve of Mathewson et al. (1992) to derive the baryonic and dark matter distributions in a sample of galaxies.

The paper is structured as follows. In Sect. 2 we present the data we used and the reduction process, then in Sect. 3 we describe the way we analysed the data. The main results are shown in Sect. 4, and then in Sect. 5 we draw our conclusions.

\section{Data acquisition and reduction}

\subsection{Photometry data}

The $B V I$ frames were taken at La Silla using the NTT telescope (New Technology Telescope), mounted with the SUSI-2 detector (D'Odorico et al. 1998), during the night of 3 to 4 February 2002. Ten frames were taken in each band, with exposures of $60 \mathrm{~s}$ ( $B$-band) and 30 seconds ( $V$ and $I$ bands). The frames were cleaned of cosmic ray artefacts, de-biased, flat-fielded, and sky-subtracted with the MIDAS software package. The images were then aligned and combined together. For flux calibration, the extinction coefficients and zero points were determined using Landolt standard star fields SA98 and SA104(b). The images of these star fields were reduced like the $B V I$ photometric images. Sky subtraction is automatically performed when the magnitude is determined in MIDAS. A flux-calibration check was done by comparing measured fluxes with data found using the Aladin sofware package (Bonnarel et al. 2000). Finally, an astrometric calibration was done using the Gaia package from Starlink.

The $H$ - and $K$ s-band images were made with the infrared imager and spectrometer SOFI (Son OF Isaac, Moorwood et al. 1998). Ten frames were taken through the $H$-filter and ten frames through the $K$ s-filter, with an exposure time of ten seconds. Between each frame, an offset frame was taken to determine the sky background. The infrared sky background varies rapidly, so following the SOFI manual, the sky background images were made using a jitter template from which a median background was determined and subtracted from the NGC 3223 frames.
Flat-fielding and illumination correction were done using the illumination and correction fields for the $H$ and $K$ s bands downloaded from the ESO SOFI website page. Flux was calibrated using the standard starfields SJ9111 and SJ9144, from which the zero point and extinction coefficients were determined using a growth curve and SNR analysis. As for the BVI frames, an astrometric calibration was performed with the Gaia package from Starlink.

\subsection{Spectroscopic data}

The spectroscopic observations were executed during 6 and 7 January 2012, for a total exposure time of $6.5 \mathrm{~h}$. Long-slit spectra were taken along the major (PA $51 \mathrm{deg}$ ) and the minor axis (-39 deg) of NGC 3223 with the FORS (FOcal Reducer and low dispersion Spectrograph, Appenzeller et al. 1998) detector mounted on the VLT telescope using a 0.7 arcsec wide slit in combination with the Gris_1028z grism. This resulted in an instrumental velocity dispersion of $\sim 20 \mathrm{~km} \mathrm{~s}^{-1}$. The covered wavelength range is 7663-9335 $\AA$, while the seeing fluctuated around 0.85 arcsec. Standard data reduction was performed with MIDAS. It included bias subtraction, flat-fielding, cosmic ray removals and wavelength calibration. Finally, the spectra were aligned and averaged, and sky subtraction was performed. The 2D error spectra were computed by taking the gain and the read out noise of the detector into account.

For the data analysis, we used ULySS ${ }^{1}$ (Koleva et al. 2009). UlySS is a full spectrum fitting package that fits the observed spectrum against a linear combination of single stellar population models, parametrised by their ages and metallicities. It takes the extinction and the imperfections of the flux calibration (or lack thereof) into account by including multiplicative polynomial to the fit. In this case we used a sixth-degree polynomial. As base models, we used Vazdekis single stellar population (SSP) models (Vazdekis et al. 2010) coupled with the CaT library from Cenarro et al. (2001). Those were convolved with the line-of-sight velocity distribution function (LOSVD). Thus, the free parameters in the fit were the age and metallicity of the SSPs, the velocity, and the velocity dispersion of the LOSVD. We used the wavelength range between $8300 \AA$ and $8680 \AA$, which is relatively clear of sky lines.

To access the instrumental broadening and the precision of the wavelength calibration we used ULySS to fit Gaussians to the reduced lamp calibration image. The instrumental dispersion that we derived changes from $25 \mathrm{~km} \mathrm{~s}^{-1}$ at $7900 \AA$ to $15 \mathrm{~km} \mathrm{~s}^{-1}$ at $9200 \AA$. This instrumental dispersion corresponds roughly to the $22.5 \mathrm{~km} \mathrm{~s}^{-1}$ broadening of the SSP models, so we could directly use the output of the program as a measure of the galaxy's physical velocity dispersion. The precision of the wavelength calibration was estimated to be around $1 \mathrm{~km} \mathrm{~s}^{-1}$. For the radial profile analysis, we binned the spectra to a minimum mean signal-to-noise of 15 , except for the outer points where we lowered our requirements to a signal-to-noise ratio of 5 .

\subsection{HI data}

The HI observations were performed at the Australia Telescope Compact Array (ATCA) with three different array setups: the $6 \mathrm{~km}$ configuration on 11 May 2002, the $1.5 \mathrm{~km}$ configuration on 21-22 July 2002, and the $750 \mathrm{~m}$ configuration on 5-6 August 2002 , for a total of $\sim 1700 \mathrm{~min}$ on source. The

htttp://ulyss.univ-lyon $1 . \mathrm{fr}$ 


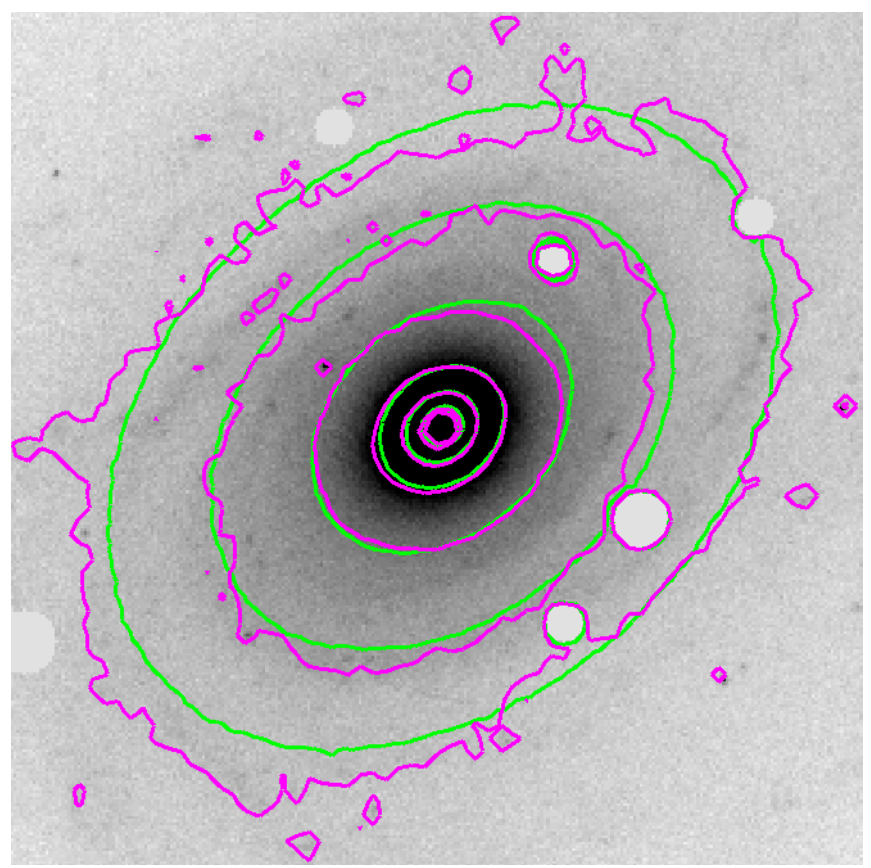

Fig. 1. Isophotes of the Ks-band image of NGC 3223 (cyan contours) fitted with FitSKIRT (green contours).

correlator setup was chosen to have $51216 \mathrm{kHz}$ channels with a total bandwidth of $8 \mathrm{MHz}$.

The data were reduced using the Miriad software package (Sault et al. 1995). We used standard procedures to flag, calibrate, and subtract the continuum. The uv-data were then Fourier inverted (using a Gaussian taper with a width in the image plane of 10 arcsec and a "robust" parameter of 0.5), and we obtained a data cube. The "dirty" cube was Hanningsmoothed, yielding a velocity resolution of $6.6 \mathrm{~km} \mathrm{~s}^{-1}$ and an rms noise of $1.2 \mathrm{mJy}^{\text {beam }}{ }^{-1}$ (corresponding to a column density of $1 \times 10^{19}$ atoms $\mathrm{cm}^{-2}$ ). We then CLEANed the data cube and restored it with a Gaussian beam of FWHM $36.94 \times$ 23.59 arcsec.

\section{Data analysis}

\subsection{Photometry}

The BVIHKs images were further analysed using the software packages FitSKIRT (which uses genetic algorithms to fit optical and NIR images, including radiative transfer calculations, De Geyter et al. 2013), and BUlge Disc Decomposition Analysis (BUDDA, de Souza et al. 2004, which was designed to perform 2D bulge-disk decompositions of optical and NIR images). We performed a 2D bulge-disk decomposition of the $K$ s-band image because it traces best the stellar disk mass. We modelled our image as a Sérsic bulge plus an exponential disk.

The fitted isophotes with FitSKIRT are shown in Fig. 1; the best-fit effective radius of the bulge is $4 \pm 0.5$ arcsec with a Sérsic index of $n=1.4 \pm 0.2$, and the best-fit exponential scale-length of the disk is $18 \pm 2$ arcsec. We double-checked this value using BUDDA and we found a consistent result. The derived apparent $K$ s-band magnitudes of the disk and bulge are $7.85 \pm 0.08$ and $9.78 \pm 0.08$, respectively. The derived inclination angle (averaged over the five $B V I H K$ s bands) is $44.1 \pm 0.5^{\circ}$.

\subsection{Stellar kinematics}

One of the main goals of the present study is to determine the stellar velocity ellipsoid, thus we have to take advantage of our observations of stellar kinematics along the major and minor axes, mostly following Gerssen \& Shapiro Griffin (2012, and references therein). Simple geometrical considerations lead to the following relation between the observed stellar velocity dispersions along the major and minor axes $\left(\sigma_{\text {maj }}\right.$ and $\sigma_{\text {min }}$, respectively) and the three components of the velocity ellipsoid:

$\sigma_{\text {maj }}^{2}=\sigma_{\theta}^{2} \sin ^{2} i+\sigma_{z}^{2} \cos ^{2} i$

$\sigma_{\min }^{2}=\sigma_{R}^{2} \sin ^{2} i+\sigma_{z}^{2} \cos ^{2} i$

where $\sigma_{R}, \sigma_{z}$, and $\sigma_{\theta}$ are the radial, vertical, and tangential components of the velocity dispersion, respectively. The inclination angle is indicated with $i$.

These are two equations and there are three unknowns, therefore a third equation needs to be added. This is usually done by assuming the epicycle approximation, which is valid in the disks of spiral galaxies because the orbits of stars are close to circular:

$\frac{\sigma_{\theta}^{2}}{\sigma_{R}^{2}}=\frac{1}{2}\left(1+\frac{\partial \ln V_{\mathrm{c}}}{\partial \ln R}\right)$

where $V_{\mathrm{c}}$ is the circular velocity of the material in the disk at a given galactocentric distance $r$. Equations (1)-(3) represent a set of three equations and (in principle) three unknowns. However, $V_{\mathrm{c}}$ is not necessarily exactly equal to the measured tangential velocity $V_{\theta}$ because of asymmetric drift:

$$
\begin{aligned}
V_{\theta}^{2}=\frac{V_{\text {maj }}^{2}}{\sin ^{2} i}= & V_{\mathrm{c}}^{2}-\sigma_{R}^{2}\left(\frac{R}{h}-R \frac{\partial}{\partial R} \ln \sigma_{R}^{2}-\frac{1}{2}-\frac{R}{2 V_{\mathrm{c}}} \frac{\partial V_{\mathrm{c}}}{\partial R}\right) \\
& +R \frac{\partial \sigma_{R z}^{2}}{\partial z}
\end{aligned}
$$

where $h$ is the exponential scale length of the stellar disk and $\sigma_{R z}^{2}$ is the tilt term, which can be safely neglected in these observations of external galaxies (Gerssen et al. 1997, 2000).

If no gas kinematics are present, the four Eqs. (1)-(4) are used to determine the four unknowns $\sigma_{R}, \sigma_{z}$ and $\sigma_{\theta}$, and $V_{\mathrm{c}}$. In our case, we have two gas kinematics observations: 2D H $\alpha$ kinematics from Palunas \& Williams (2000) and our own HI data (radially more extended but with a lower angular resolution). We use the $\mathrm{H} \alpha$ data as an extra piece of information and as a consistency check for the circular velocities determined from stellar kinematics.

However, direct inversion of the equations to determine the unknowns as a function of radius results in very large uncertainties due to error propagation and quite noisy data to start with. Therefore we followed the approach taken by e.g. Gerssen \& Shapiro Griffin (2012, and references therein), where the radial and vertical components of the velocity dispersion are modelled as exponentials (in the radial direction),

$$
\begin{gathered}
\sigma_{R}=\sigma_{R, 0} \mathrm{e}^{-R / a_{R}} \\
\sigma_{z}=\sigma_{z, 0} \mathrm{e}^{-R / a_{z}}
\end{gathered}
$$

and the circular velocity as a function of radius (the rotation curve) is modelled as a power law:

$V_{\mathrm{c}}=V_{0} R^{\alpha}$ 
Hence the problem reduces to modelling the observed $\sigma_{\text {maj }}(r)$, $\sigma_{\text {min }}(r), V_{\text {maj }}(r)$, and gas rotation curve with six parameters $\left(\sigma_{R, 0}, \sigma_{z, 0}, a_{R}, a_{z}, V_{0}\right.$, and $\left.\alpha\right)$.

The central parts of the galaxy are clearly dominated by the bulge. Therefore, in our analysis we only consider the radii where the disk-to-bulge brightness ratio is larger than 20. Based on the disk and bulge parameters derived in Sect. 3.1, we thus only consider radii larger than $3.2 \mathrm{kpc}$ (17.3 arcsec).

In a locally isothermal disk the vertical density distribution is given by $\operatorname{sech}^{2}\left(z / z_{0}\right)$ where $z$ is the vertical distance from midplane and $z_{0}$ is the vertical scale-height. In this case, the disk surface density $\Sigma$, and the scale-height $z_{0}$ are linked to the vertical velocity dispersion through the following relation:

$\sigma_{z}^{2}=\pi G \Sigma z_{0}$

As shown by e.g. Bottema (1993), if the disk $M / L$ ratio is constant with radius, then the equation above can be rewritten as follows:

$\sigma_{z}^{2}=\pi G(M / L) I_{K_{\mathrm{s}}} z_{0}$

and $I_{K_{\mathrm{s}}}=I_{0} \mathrm{e}^{-R / h}$ is the surface brightness of the stellar disk (in the $K$ s-band) measured here. Assuming the kinematic scalelength (for the vertical velocity dispersion) is twice the photometric scale-length, i.e. $a_{z}=2 h$ (Bottema 1993; Binney \& Merrifield 1998), we can rewrite Eq. (9) to get an independent estimate of the $M / L$ ratio:

$(M / L)=\frac{\sigma_{z, 0}^{2}}{\pi G I_{0} z_{0}}$.

In our analysis, we investigate models where $a_{z}=2 h, a_{z}=h$, and $a_{z}$ is a free parameter.

\subsection{HI data cube analysis}

Using the primary beam-corrected data cube, we find a total HI flux of $26.6 \mathrm{Jy} \mathrm{km} \mathrm{s}{ }^{-1}$, corresponding (at a distance of $38.1 \mathrm{Mpc}$ ) to $9.1 \times 10^{9} M_{\odot}$. The total HI map is superimposed onto an optical image in Fig. 2.

Thorough modelling of the data cube of NGC 3223 goes beyond the aim of this paper, but we made some simple models to have a good understanding of the HI distribution and kinematics. We used the code called TiRiFiC (Józsa et al. 2007) to make models of the HI data cube, which we iteratively compared to the observed one (see Gentile et al. 2013 for more details about the procedure). The best-fit model is shown in Fig. 3: it gives a very good representation of the observations. The data cube, Fig. 3 and the total HI map indicate that NGC 3223 has a regularly rotating HI disk, see the velocity field shown in Fig. 4. The gaseous disk also has an extension to the SE around position (10 2145 , -3417 30), see Fig. 2. This extension is present in the channel maps between velocities 2678 and $2704 \mathrm{~km} \mathrm{~s}^{-1}$.

Particular attention was given to the derivation of the rotation curve. A first estimate was derived from the tilted-ring fit of the intensity-weighted velocity field, then the rotation curve was iteratively modified to match the observed data cube, in particular the position-velocity diagram along the major axis. Each data point is assigned an uncertainty that is the maximum between two quantities: (1) the difference between the two sides (receding and approaching); and (2) the velocity resolution corrected for inclination. The best-fit inclination in the inner parts $\left(52^{\circ}\right.$, but with an uncertainty of about $10^{\circ}$ because of the relatively irregular shape of the HI contours) is slightly higher

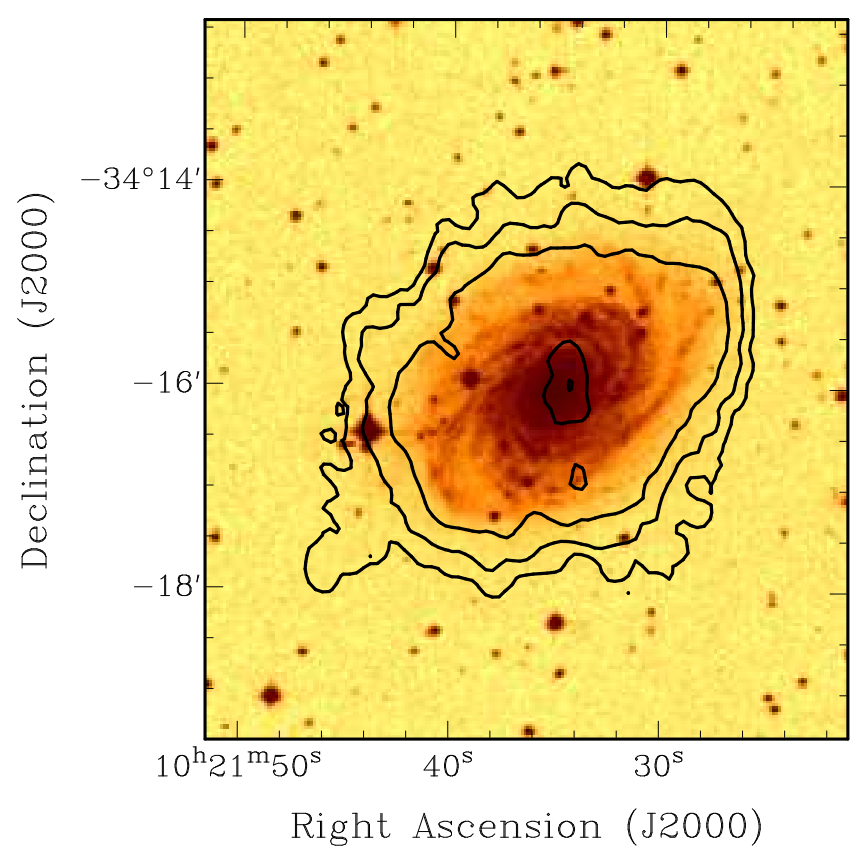

Fig. 2. Total HI map of NGC 3223 (contours) overlaid onto an optical DSS image (colour scale). Contour levels are $(1.5,3,6,12) \times$ $10^{20}$ atoms $\mathrm{cm}^{-2}$. Note that the central contour represents a drop in HI surface density.

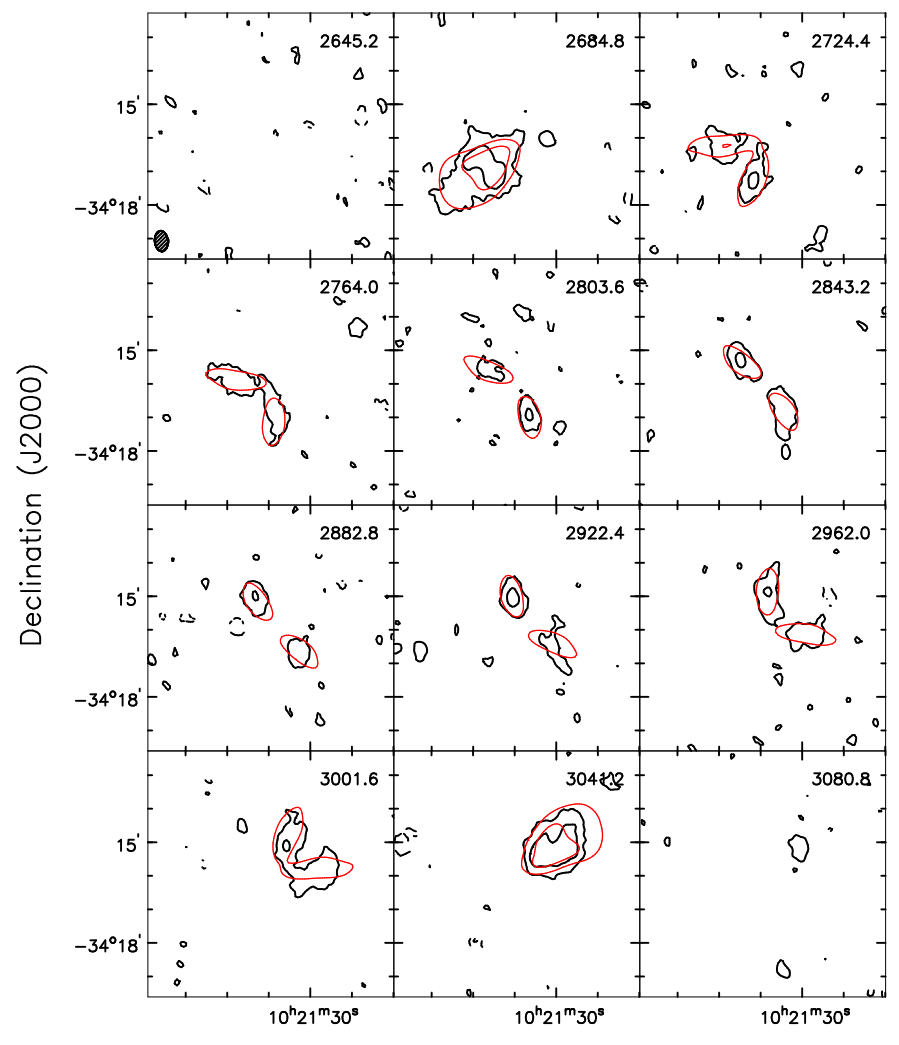

Right Ascension (J2000)

Fig. 3. Hi data cube of NGC 3223. The beam is shown in the bottom left of the top left panel $(36.9 \times 23.6$ arcsec $)$. The contours are $-3,3(2.5 \sigma)$, and $9 \mathrm{mJy}$ beam $^{-1}$. Negative contours are dashed. The observed data cube is shown as thick black contours and the model presented here is shown as thin red contours.

than the one derived from the optical and NIR data $\left(44.1^{\circ}\right.$, see Sect. 3.1). When plotting the two rotation curves together, the 
Table 1. Best-fit parameters of the joint $\sigma_{\text {min }}-\sigma_{\text {maj }}-V_{\text {rot }}-V_{\mathrm{c}}$ fit.

\begin{tabular}{lllllll}
\hline \hline Fit & $\begin{array}{l}\sigma_{R, 0} \\
\mathrm{~km} \mathrm{~s}^{-1}\end{array}$ & $\begin{array}{l}\sigma_{z, 0} \\
\mathrm{~km} \mathrm{~s}^{-1}\end{array}$ & $\begin{array}{l}a_{R} \\
\mathrm{kpc}\end{array}$ & $\begin{array}{l}a_{z} \\
\mathrm{kpc}\end{array}$ & $\begin{array}{l}V_{0} \\
\mathrm{~km} \mathrm{~s}^{-1}\end{array}$ & $\alpha$ \\
\hline All free & $182 \pm 29$ & $140 \pm 16$ & $5.1 \pm 1.3$ & $12.7 \pm 3.4$ & $280.6 \pm 1.5$ & $0.064 \pm 0.009$ \\
$a_{R}=a_{z}$ & $135 \pm 12$ & $162 \pm 12$ & $8.9 \pm 0.8$ & $=a_{R}$ & $284.5 \pm 1.8$ & $0.097 \pm 0.014$ \\
$a_{z}=2 h$ & $129 \pm 15$ & $192 \pm 11$ & $10.2 \pm 1.8$ & 6.6 (fixed) & $285.9 \pm 1.9$ & $0.102 \pm 0.014$ \\
$a_{z}=2 a_{R}=2 h$ & $232 \pm 13$ & $222 \pm 8$ & 3.3 (fixed) & 6.6 (fixed) & $275.8 \pm 1.2$ & $0.053 \pm 0.007$ \\
\hline
\end{tabular}

Notes. The stellar exponential scale-length $h$ is $18 \pm 2$ arcsec, corresponding to $3.3 \pm 0.4 \mathrm{kpc}$.

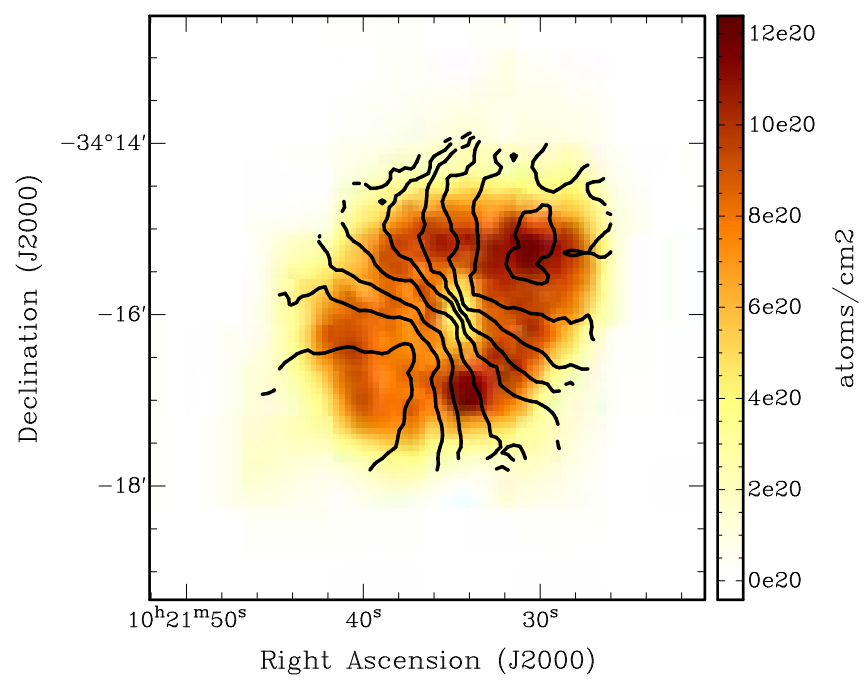

Fig. 4. Intensity-weighted HI velocity field of NGC 3223 (contours) overlaid onto the total HI map (colour scale). Contour levels start from $2704 \mathrm{~km} \mathrm{~s}^{-1}$ in steps of $40 \mathrm{~km} \mathrm{~s}^{-1}$.

HI data points were corrected for the inclination difference; we consider the inclination from the optical and NIR data to be more reliable because of the less regular shape of the HI emission.

\section{Results}

The first fit we made was with the six parameters $\left(\sigma_{R, 0}, \sigma_{z, 0}\right.$, $a_{R}, a_{z}, V_{0}$, and $\alpha$ ) described in Sect. 3.2, fitting simultaneously the major axis stellar velocity dispersion profile, the minor axis stellar velocity dispersion profile, the major axis stellar rotation velocity profile, and the circular velocity curve as derived from the gas kinematics (from the $\mathrm{H} \alpha$ data - the HI data were used for the mass decomposition fits but not for the combined velocity dispersion/circular velocity fits). The fit quality is very good but some parameters are rather weakly constrained. The results of all the fits are tabulated in Table 1, and the fits are plotted in Fig. 5.

To investigate the real uncertainties on the parameters in a better way, we then made a fit where we forced the two kinematic scale-lengths (radial and vertical, $a_{r}$ and $a_{z}$ respectively) to have the same value, as in Shapiro et al. (2003) and Gerssen $\&$ Shapiro Griffin (2012). The next choice was to impose $a_{z}=2 h$ in order to be able to use Eq. (10) to estimate the stellar $M / L$ ratio. Finally, we made a fit with only four free parameters $\left(\sigma_{R, 0}\right.$, $\sigma_{z, 0}, V_{0}$, and $\alpha$ ) and fixing $a_{R}=h$ (to have a constant Toomre stability parameter with radius) and $a_{z}=2 h$ (like above, to obtain an estimate of the stellar $M / L$ ratio). The uncertainties on the fitted parameters were derived by creating 100 randomly generated data sets from Gaussian distributions defined by the observed data. This means for each realisation we generated a new data point, corresponding to each original data point, by randomly sampling from the original point with a Gaussian distribution with variance equal to our originally computed variance. These new data points were assumed to have the same variance as the originals. These randomly generated data were then subjected to the same fitting procedure ( $\chi^{2}$ minimisation) as the original data, which gives distributions of values for the best-fit parameters and hence estimates of their $1 \sigma$ uncertainties from Gaussian fits of their distribution.

\subsection{Disk heating}

From observations of eight spiral galaxies, Gerssen \& Shapiro Griffin (2012) find a relation between Hubble type and $\sigma_{z} / \sigma_{R}$ ratios: it turns out that later type galaxies have lower $\sigma_{z} / \sigma_{R}$ ratios. From the relation they derive, the expected value for an Sb galaxy like NGC 3223 would be around 0.63 with an uncertainty of about 0.2 . From the fit with $a_{r}=a_{z}$ we find $\sigma_{z} / \sigma_{R}=1.21 \pm 0.14$, which is not consistent with Gerssen's relation. If we force the ratio to be equal to 0.63 , we find (Fig. 5) that the fit indeed worsens, in particular the major axis velocity dispersion and rotation velocity profiles are underestimated.

It is possible that we overestimated the inclination $\left(44.1^{\circ}\right)$. By taking $40.5^{\circ}$, the value of Kassin et al. (2006a), we find a best-fit ratio of $\sigma_{z} / \sigma_{R}=1.28 \pm 0.17$, which is still higher than the value suggested by Gerssen \& Shapiro Griffin (2012). It is also possible that the choice of cutoff radius is incorrect. Changing the inner cutoff radius to 15 arcsec (instead of 17.3 arcsec) gives an almost identical value, $\sigma_{z} / \sigma_{R}=1.19 \pm 0.11$. Changing it to 25 arcsec gives $\sigma_{z} / \sigma_{R}=1.07 \pm 0.19$, slightly lower but (as intuitively expected) with a larger uncertainty, because there are fewer data points to constrain the parameters.

The result that NGC 3223 is more vertically heated than expected implies that NGC 3223 is an outlier in the observational relation of Gerssen \& Shapiro Griffin (2012). The reason for this large vertical heating is not obvious, but recent mergers are excluded because of the regular morphology and kinematics. We note that the values we found for NGC 3223 are marginally inconsistent with the correlation between $\sigma_{z}$ and $\sigma_{z} / \sigma_{R}$ found by Gerssen \& Shapiro Griffin (2012). They plot the velocity dispersions at half the kinematic scale-length. With $\sigma_{z}=98.3 \pm 7.3 \mathrm{~km} \mathrm{~s}^{-1}$ and $\sigma_{z} / \sigma_{R}=1.21 \pm 0.14$, NGC 3223 is (marginally) inconsistent with the relation found in their Fig. 6.

\subsection{Constraint on the $M / L$ ratio}

The evaluation of constraints on the stellar $M / L$ ratio was done using the results of the fits made with the scale length of the vertical velocity dispersion distribution fixed at twice the stellar exponential scale length (see Sect. 4).

Applying Eq. (10) to the fits using $a_{z}=2 \mathrm{~h}$ and $a_{z}=2 a_{R}=2 \mathrm{~h}$ in Table 1 gives values of $M / L=0.96 \pm 0.34$ and $1.28 \pm 0.46$, 


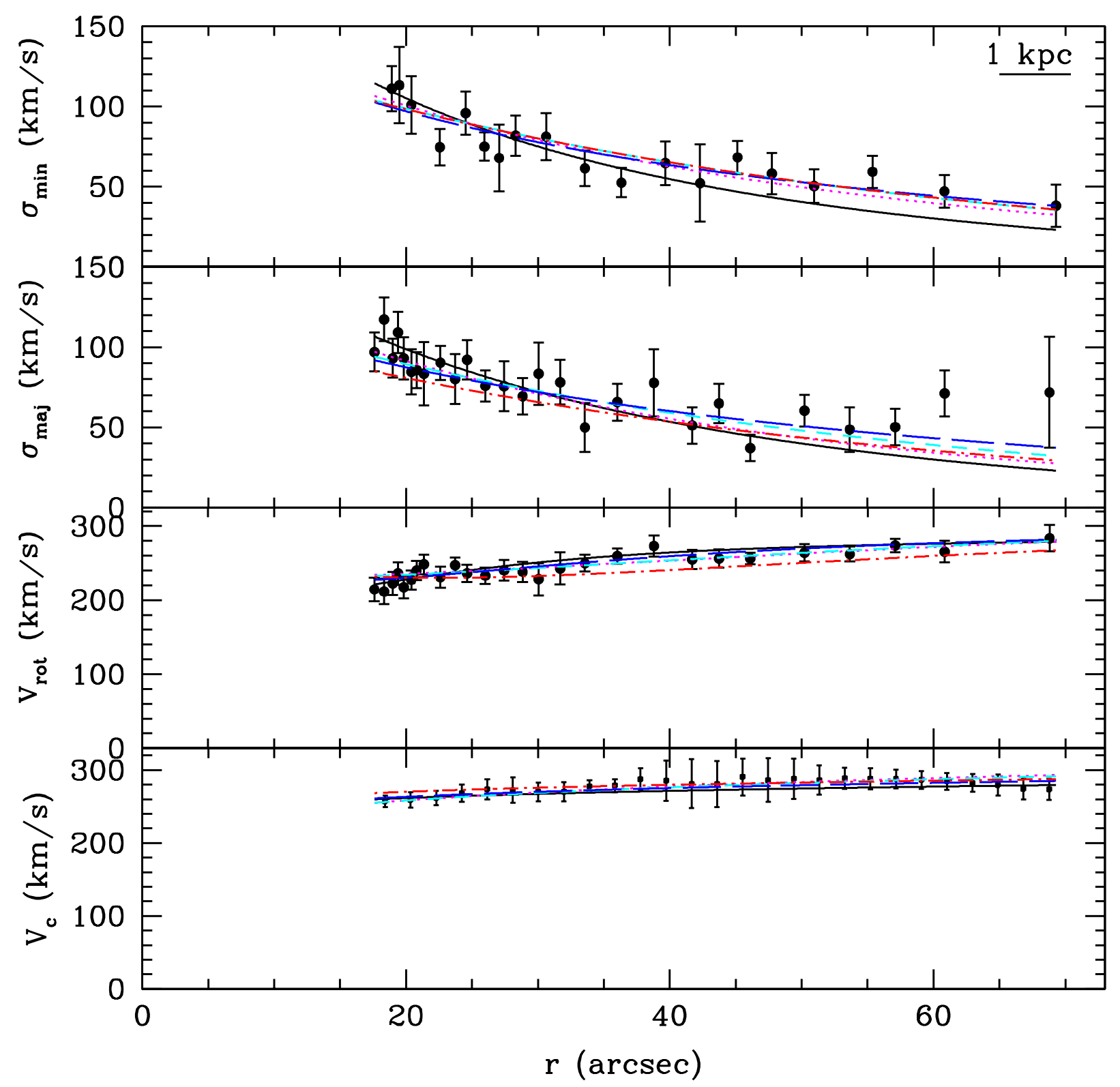

Fig. 5. Fit with all six free parameters (blue long-dashed lines), fit with $a_{z}=a_{R}$ left as a free parameter (cyan short-dashed lines), fit with $a_{z}$ fixed at $6.6 \mathrm{kpc}$ (twice the disk scale-length) and $a_{R}$ free (magenta dotted lines), fit with $a_{z}$ fixed at $6.6 \mathrm{kpc}$ (twice the disk scale-length) and $a_{R}$ fixed at the disk scale-length, $3.3 \mathrm{kpc}$ (black solid lines), and fit it with $a_{z}=a_{R}$ left as a free parameter, and with $\sigma_{z} / \sigma_{R}$ fixed at 0.63 (red dot-dashed lines). Note that the bottom panel has a different horizontal scale from the top three panels. In the bottom panel the $\mathrm{H} \alpha$ data (full circles) come from Palunas \& Williams (2000).

respectively, which is quite high for the $K$ s-band. The errors quoted here are formal uncertainties based on the fits and error propagation. We used a $\operatorname{sech}^{2}$ vertical distribution with a scaleheight of $0.9 \mathrm{kpc}$, based on the $K \mathrm{~s}$-band exponential scale-length and using the average between the scale-length-to-scale-height ratios given by Kregel et al. (2002) and Bershady et al. (2010b). We used the $K$ s-band absolute magnitude of the Sun given by Pecaut \& Mamajek (2013), i.e. 3.30.

Could other effects contribute to the real uncertainties? One possibility is the inclination angle: if we assume $40.5^{\circ}$ (see previous section) the results above become $0.91 \pm 0.33$ and $1.08 \pm 0.39$, respectively. Changing the inner cutoff radius to 15 arcsec instead of 17.3 arcsec gives (for $a_{z}=2 h$ and $a_{z}=2 a_{R}=2 h$ ) $0.98 \pm$ 0.35 and $1.25 \pm 0.45$, thus a negligible variation in the derived stellar $M / L$ ratio. Changing the inner cutoff radius to $25 \operatorname{arcsec}$ instead of $17.3 \operatorname{arcsec}$ gives $0.75 \pm 0.27$ and and $1.36 \pm 0.49$, respectively.

The expectations from stellar population synthesis range from 0.35 to 0.80 , using a $B-V$ colour of 0.80 and the relations between colour and stellar $M / L$ ratio found in Bell \& de Jong (2001), Bell et al. (2003), Portinari et al. (2004), Zibetti et al. (2009), and Into \& Portinari (2013). The B-V colour seems to be a good indicator of $M / L$ ratio (McGaugh \& Schombert 2014), even though at NIR wavelengths the dependency of the $M / L$ ratio on colour is weak.

Therefore we can conclude that our estimated range of stel$\operatorname{lar} M / L$ ratios (between 0.5 and 1.7) is consistent with the most massive predictions of stellar population synthesis models. We have checked that within the outermost point fitted in the velocity dispersion profiles the stellar surface density (even with 

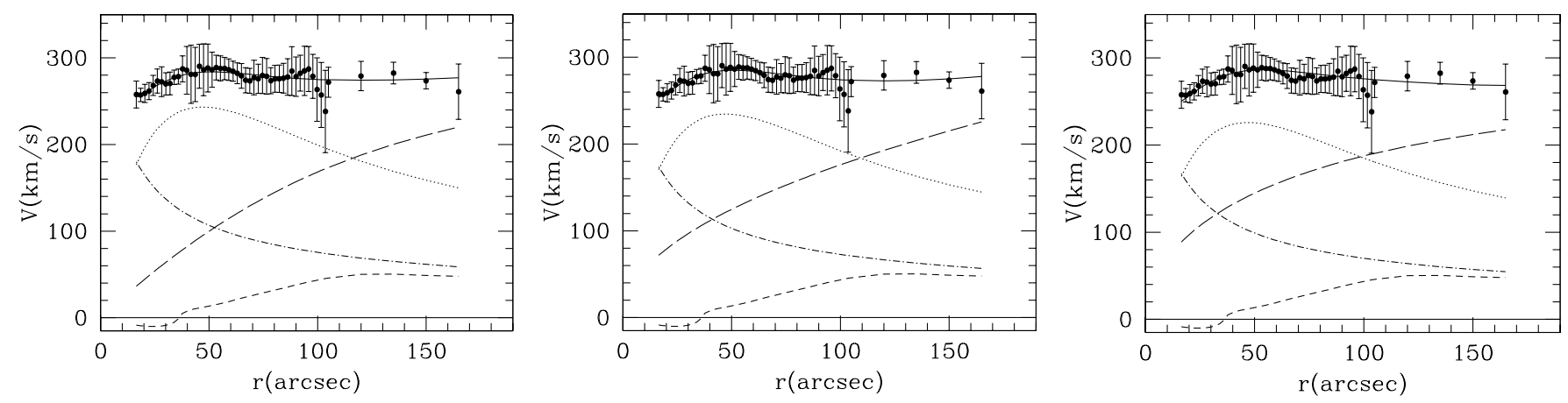

Fig. 6. Rotation curve fits. Left panel: Burkert halo, middle: unconstrained NFW halo; right panel: NFW halo using the $c-M_{\mathrm{vir}}$ relation. The points with errorbars represent the combined $\mathrm{H} \alpha+\mathrm{HI}$ rotation curve. The short dashed, long dashed, dotted, and dot-dashed curves represent the gas, halo, stellar disk, and bulge contributions, respectively. The total (best) fit to the rotation curve for a particular model is represented by a solid line.

Table 2. Best-fit parameters of the rotation curve fits.

\begin{tabular}{llll}
\hline \hline Fit & Stellar $M / L_{K}$ ratio & Parameter 1 & Parameter 2 \\
\hline Burkert halo & $0.71_{-0.04}^{+0.04}$ & $26.9_{-10.5}^{+31.5}$ & $0.0087_{-0.0040}^{+0.0075}$ \\
Unconstrained NFW halo & $0.66_{-0.08}^{+0.03}$ & $10545_{-10510}^{+5530}$ & $1_{-0}^{+9.6}$ \\
NFW halo with $c-M_{\text {vir }}$ relation & $0.61_{-0.02}^{+0.02}$ & $57_{-18}^{+22}$ & $8.0_{-0.4}^{+0.5}$ \\
\hline
\end{tabular}

Notes. For the Burkert halo "Parameter 1" is the core radius in kpc and "Parameter 2" is the central density in $M_{\odot} \mathrm{pc}^{-3}$, whereas for the NFW halo "Parameter 1" is the virial mass in $10^{11} M_{\odot}$ and "Parameter 2" is the concentration.

a stellar $M / L$ ratio of 0.5 ) is much higher ( $>10$ times) than the HI surface density: thus our results about the $M / L$ ratio are not falsified by the HI surface density influencing the vertical velocity dispersion.

\subsection{Rotation curve fitting}

Using the constraints on the stellar $M / L$ ratio derived in Sect. 4.2 we made rotation curve fits using different dark matter halo density distributions. The bulge and disk contributions to the rotation curve were derived from the parameters of the $K$ s-band bulge/disk decomposition of Sect. 3.1. For the bulge we adopt the same stellar $M / L$ ratio as for the disk. The HI disk contribution was derived from the observed surface density distribution (corrected for primordial He), and for the dark matter halo we used two different functional forms:

- the Burkert (1995) halo, known to give good fits to rotation curves (e.g. Salucci et al. 2007). The density $\rho(R)$ as a function of radius $R$ has a central core (i.e. a finite central density) and is given by:

$\rho_{\text {Bur }}(R)=\frac{\rho_{0} R_{\text {core }}^{3}}{\left(R+R_{\text {core }}\right)\left(R^{2}+R_{\text {core }}^{2}\right)}$

where the central density $\rho_{0}$ and the core radius $R_{\text {core }}$ are the two free parameters.

- the NFW halo (Navarro et al. 1996), characterised by a central density cusp, whose functional form is the following:

$\rho_{\mathrm{NFW}}(R)=\frac{\rho_{\mathrm{s}}}{\left(R / R_{\mathrm{S}}\right)\left(1+R / R_{\mathrm{S}}\right)^{2}}$,

where $\rho_{\mathrm{s}}$ and $R_{\mathrm{s}}$ are the characteristic density and scalelength of the dark matter halo. The parameters $\rho_{\mathrm{s}}$ and $R_{\mathrm{S}}$ can also be expressed in terms of the virial mass $M_{\text {vir }}$ and the concentration $c=R_{\mathrm{vir}} / R_{\mathrm{s}}\left(R_{\mathrm{vir}}\right.$ is the virial radius). Dark matter simulations of structure formation in the Universe show that $c$ and $M_{\text {vir }}$ are not independent (e.g. Neto et al. 2007). We adopt here the $c-M_{\text {vir }}$ relation used by Gentile et al. (2007):

$c_{\mathrm{vir}} \simeq 13.6\left(\frac{M_{\mathrm{vir}}}{10^{11} M_{\odot}}\right)^{-0.13}$.

The fits are plotted in Fig. 6: the Burkert halo fit is very good, and the product $\rho_{0} R_{\text {core }}=232_{-133}^{+338} M_{\odot} \mathrm{pc}^{-2}$ is consistent with the value found by Donato et al. (2009) and Gentile et al. (2009) for a large sample of galaxies.

The NFW halo fits are almost as good. In the fit with no constraints on the virial mass and concentration the allowed range of virial masses spans almost three orders of magnitude. This range would have been larger if we had not put a minimum reasonable value of 1 for the concentration parameter. The constrained NFW halo fit is only slightly worse $\left(\chi^{2}\right.$ value $\sim 35 \%$ higher $)$, we find a value of the virial mass of $5.6_{-1.7}^{+2.1} \times 10^{12} M_{\odot}$, consistent with the value of the unconstrained fit.

\section{Conclusions}

We have presented observations of the stars and gas kinematics of NGC 3223, an Sb galaxy located at a distance of about $38 \mathrm{Mpc}$.

From the observed stellar velocity dispersion along the major and minor axis, from the major-axis stellar rotation velocity and from the gas kinematics, we determined the vertical and radial stellar velocity dispersion as a function of radius. We found a vertical-to-radial velocity dispersion ratio of $\sigma_{z} / \sigma_{R}=$ $1.21 \pm 0.14$, significantly higher the relation between $\sigma_{z} / \sigma_{R}$ and Hubble type found by e.g. Gerssen \& Shapiro Griffin (2012). 
We also used the $K$ s-band photometry together with the derived vertical velocity dispersion to estimate the $M / L$ ratio of the stellar disk. We find that the uncertainties are quite large, and the allowed range lies between 0.5 and 1.7 (in the $K$ s-band). This lies at the high end of the values predicted by stellar population synthesis models. Because of such a weak constraint on the stellar $M / L$ ratio we cannot really constrain the distribution of dark matter in NGC 3223. However, the method proved to yield meaningful results, consistent with other data, therefore a statistical approach involving a sample of galaxies with similar data and a spread in physical properties will certainly provide more answers.

Acknowledgements. We thank the anonymous referee for constructive comments that improved the content and presentation of this paper. The research of GWA supported by the FWO - Vlaanderen. M.K. is a postdoctoral fellow of the Fund for Scientific Research-Flanders, Belgium (FWO 65052/12E4115N LV).

\section{References}

Appenzeller, I., Fricke, K., Fürtig, W., et al. 1998, The Messenger, 94, 1 Bell, E. F., \& de Jong, R. S. 2001, ApJ, 550, 212

Bell, E. F., McIntosh, D. H., Katz, N., \& Weinberg, M. D. 2003, ApJS, 149, 289 Bershady, M. A., Verheijen, M. A. W., Swaters, R. A., et al. 2010a, ApJ, 716, 198

Bershady, M. A., Verheijen, M. A. W., Westfall, K. B., et al. 2010b, ApJ, 716, 234

Binney, J., \& Merrifield, M. 1998, Galactic astronomy (Princeton, NJ: Princeton University Press)

Bonnarel, F., Fernique, P., Bienaymé, O., et al. 2000, A\&AS, 143, 33

Bosma, A. 1981, AJ, 86, 1825

Bottema, R. 1993, A\&A, 275, 16

Bottema, R. 1997, A\&A, 328, 517

Burkert, A. 1995, ApJ, 447, L25

Candlish, G. N., Smith, R., Fellhauer, M., et al. 2014, MNRAS, 437, 3702

Cenarro, A. J., Cardiel, N., Gorgas, J., et al. 2001, MNRAS, 326, 959

Cloet-Osselaer, A., De Rijcke, S., Schroyen, J., \& Dury, V. 2012, MNRAS, 423, 735

Courteau, S., Cappellari, M., de Jong, R. S., et al. 2014, Rev. Mod. Phys., 86, 47 de Souza, R. E., Gadotti, D. A., \& dos Anjos, S. 2004, ApJS, 153, 411 de Blok, W. J. G., McGaugh, S. S., \& Rubin, V. C. 2001, AJ, 122, 2396 de Blok, W. J. G., Walter, F., Brinks, E., et al. 2008, AJ, 136, 2648 De Geyter, G., Baes, M., Fritz, J., \& Camps, P. 2013, A\&A, 550, A74 de Jong, R. S., \& Bell, E. F. 2007, Island Universes - Structure and Evolution of Disk Galaxies, 107

Di Cintio, A., Brook, C. B., Macciò, A. V., et al. 2014, MNRAS, 437, 415

D'Odorico, S., Beletic, J. W., Amico, P., et al. 1998, Proc. SPIE, 3355, 507
Donato, F., Gentile, G., Salucci, P., et al. 2009, MNRAS, 397, 1169

Fuchs, B. 2003, Ap\&SS, 284, 719

Gentile, G., Salucci, P., Klein, U., Vergani, D., \& Kalberla, P. 2004, MNRAS, 351,903

Gentile, G., Salucci, P., Klein, U., \& Granato, G. L. 2007, MNRAS, 375, 199

Gentile, G., Famaey, B., Zhao, H., \& Salucci, P. 2009, Nature, 461, 627

Gentile, G., Józsa, G. I. G., Serra, P., et al. 2013, A\&A, 554, A125

Gerssen, J., \& Shapiro Griffin, K. 2012, MNRAS, 423, 2726

Gerssen, J., Kuijken, K., \& Merrifield, M. R. 1997, MNRAS, 288, 618

Gerssen, J., Kuijken, K., \& Merrifield, M. R. 2000, MNRAS, 317, 545

Governato, F., Zolotov, A., Pontzen, A., et al. 2012, MNRAS, 422, 1231

Hänninen, J., \& Flynn, C. 2002, MNRAS, 337, 731

Into, T., \& Portinari, L. 2013, MNRAS, 430, 2715

Jenkins, A., \& Binney, J. 1990, MNRAS, 245, 305

Józsa, G. I. G., Kenn, F., Klein, U., \& Oosterloo, T. A. 2007, A\&A, 468, 731

Kassin, S. A., de Jong, R. S., \& Pogge, R. W. 2006a, ApJS, 162, 80

Kassin, S. A., de Jong, R. S., \& Weiner, B. J. 2006b, ApJ, 643, 804

Kazantzidis, S., Zentner, A. R., Kravtsov, A. V., Bullock, J. S., \& Debattista, V. P. 2009, ApJ, 700, 1896

Koleva, M., Prugniel, P., Bouchard, A., \& Wu, Y. 2009, A\&A, 501, 1269

Kregel, M., van der Kruit, P. C., \& de Grijs, R. 2002, MNRAS, 334, 646

Kregel, M., van der Kruit, P. C., \& Freeman, K. C. 2005, MNRAS, 358, 503

Kroupa, P. 2002, MNRAS, 330, 707

Kuzio de Naray, R., \& Kaufmann, T. 2011, MNRAS, 414, 3617

Macciò, A. V., Stinson, G., Brook, C. B., et al. 2012, ApJ, 744, L9

Martinsson, T. P. K., Verheijen, M. A. W., Westfall, K. B., et al. 2013, A\&A, 557, A131

Mathewson, D. S., Ford, V. L., \& Buchhorn, M. 1992, ApJS, 81, 413

McGaugh, S., \& Schombert, J. 2014, AJ, 148, 77

Minchev, I., \& Quillen, A. C. 2006, MNRAS, 368, 623

Moorwood, A., Cuby, J.-G., \& Lidman, C. 1998, The Messenger, 91,9

Neto, A. F., Gao, L., Bett, P., et al. 2007, MNRAS, 381, 1450

Oh, S.-H., Brook, C., Governato, F., et al. 2011a, AJ, 142, 24

Oh, S.-H., de Blok, W. J. G., Brinks, E., Walter, F., \& Kennicutt, R. C., Jr. 2011b, AJ, 141, 193

Palunas, P., \& Williams, T. B. 2000, AJ, 120, 2884

Pecaut, M. J., \& Mamajek, E. E. 2013, ApJS, 208, 9

Pérez, I., Fux, R., \& Freeman, K. 2004, A\&A, 424, 799

Persic, M., Salucci, P., \& Stel, F. 1996, MNRAS, 281, 27

Portinari, L., Sommer-Larsen, J., \& Tantalo, R. 2004, MNRAS, 347, 691

Salucci, P., Lapi, A., Tonini, C., et al. 2007, MNRAS, 378, 41

Sault, R. J., Teuben, P. J., \& Wright, M. C. H. 1995, Astronomical Data Analysis Software and Systems IV, 77, 433

Sellwood, J. A. 2014, Rev. Mod. Phys., 86, 1

Sellwood, J. A., \& Binney, J. J. 2002, MNRAS, 336, 785

Shapiro, K. L., Gerssen, J., \& van der Marel, R. P. 2003, AJ, 126, 2707

van Eymeren, J., Trachternach, C., Koribalski, B. S., \& Dettmar, R.-J. 2009, A\&A, 505, 1

Vazdekis, A., Sánchez-Blázquez, P., Falcón-Barroso, J., et al. 2010, MNRAS, 404, 1639

Weiner, B. J., Sellwood, J. A., \& Williams, T. B. 2001, ApJ, 546, 931

Zibetti, S., Charlot, S., \& Rix, H.-W. 2009, MNRAS, 400, 1181 